Hedley, G. J., Steiner, F., Vogelsang, J. and Lupton, J. M. (2018) Fluctuations in the emission polarization and spectrum in single chains of a common conjugated polymer for organic photovoltaics. Small, 14(51), 1804312.

There may be differences between this version and the published version. You are advised to consult the publisher's version if you wish to cite from it.

This is the peer reviewed version of the following article:

Hedley, G. J. , Steiner, F., Vogelsang, J. and Lupton, J. M. (2018)

Fluctuations in the emission polarization and spectrum in single chains of a common conjugated polymer for organic photovoltaics. Small, 14(51), 1804312. (doi:10.1002/smll.201804312)

This article may be used for non-commercial purposes in accordance with Wiley Terms and Conditions for Self-Archiving.

http://eprints.gla.ac.uk/172127/

Deposited on: 06 November 2018

Enlighten - Research publications by members of the University of Glasgow http://eprints.gla.ac.uk/ 


\section{Fluctuations in the Emission Polarization and Spectrum in Single Chains of a Common Conjugated Polymer for Organic Photovoltaics}

Gordon J. Hedley, * Florian Steiner, Jan Vogelsang and John M. Lupton

Dr G.J. Hedley

WestCHEM, School of Chemistry, University of Glasgow, Glasgow G12 8QQ, United Kingdom

E-mail: Gordon.Hedley@glasgow.ac.uk

Dr. G.J. Hedley, Dr F. Steiner, Prof. J.M. Lupton

Institut für Experimentelle und Angewandte Physik, Universität Regensburg, D-93040,

Regensburg, Germany

Dr F. Steiner, Dr J. Vogelsang

Department Chemie, Ludwig-Maximilians-Universität München, Butenandtstr. 5 - 13, 81377

München, Germany

Keywords: single molecule spectroscopy, organic semiconductors, nanoscale organization, spectroscopic fluctuations

Measuring the nanoscale organization of conjugated polymer chains used in organic photovoltaic (OPV) blends is vital if one wants to understand the materials. This is made very difficult with high efficiency OPV polymers such as PTB7 that form aggregates, as a lack of periodicity and a high degree of disorder make understanding of the nanoscale organization challenging. Here we use single molecule spectroscopy to observe single chains and aggregates of PTB7. Using four detectors we simultaneously monitor the photoluminescence intensity, wavelength, polarization and lifetime. We observe fast (milliseconds) and slow (seconds) fluctuations over a time window of $30 \mathrm{~s}$ in all of these observables from single aggregates and chains as individual chromophores activate and deactivate, leading to dynamical changes in the emission spectrum and dipole orientation. This information can be used to help reconstruct the spatial and spectral organization of disordered aggregates of PTB7, thereby adding valuable new information on how the chains are arranged in space. 


\section{Introduction}

Conjugated polymer based organic photovoltaic (OPV) devices now regularly achieve power conversion efficiencies above $10 \%,{ }^{[1-3]}$ with $14 \%$ efficient devices recently reported. ${ }^{[4]}$ Fundamentally, light absorption is the first step in the path to enable extraction of useful work from the device. ${ }^{[5]}$ Light is primarily absorbed by the conjugated polymer in a solar cell blend, and thus the polymer's physical and electrical properties are of significant interest as they ultimately control the device efficiency. The chain alignment, degree of aggregation and nature of the excited states play a role in light absorption, exciton diffusion to the donor-acceptor interface, charge separation and finally charge extraction. ${ }^{[5-8]}$ These properties of conjugated polymers are often hard to measure and are interdependent upon each other, such that a clear and fully understood microscopic picture is rarely achieved. Single molecule spectroscopy $(\mathrm{SMS})^{[9-12]}$ can help to address this challenge by enabling individual chains or aggregates of chains to be separated from each other, allowing the photophysics to be studied in a step-wise manner from the bottom up, ${ }^{[13-15]}$ free from the overwhelming complexity of an ensemble, where everything is contributing simultaneously to the observables. SMS can thus help to contribute to understanding of the properties of materials used in OPVs, with such understanding being a prerequisite to understanding of OPV performance.

The polymer PTB7 (chemical structure shown in the inset of Figure 1, full chemical name in Experimental section) is prototypical of the so-called "third generation" of materials in OPV, having a low observed ensemble bandgap $(\sim 1.75 \mathrm{eV})$, with chains comprising of alternating donor and acceptor units and achieving efficiencies of 7-10\%. ${ }^{[1,16,17]}$ Previously using single molecule spectroscopy (SMS) we have observed ${ }^{[18]}$ that the low bandgap in PTB7 is in fact caused by aggregation of chains, semi-disordered in nature, that leads to a significant redshifting of the emission (and by presumption absorption). The true optical gap in PTB7 was measured to be $\sim 0.4 \mathrm{eV}$ higher when straight non-aggregated polymer chains were isolated. Single molecule microscopy measurements are the only way to study such non-aggregated 
single chains without using elevated temperature solutions, ${ }^{[19,}{ }^{20]}$ as in room temperature ensemble experiment self-absorption from the redder aggregated chains in the immediate locale will make the higher energy emission impossible to see. The existence of a larger bandgap indicates just how important aggregation of the chains of PTB7 are, as it is responsible for the red-shifting of absorption, giving rise to a better match of the material with the solar spectrum. The nature and degree of these aggregated "red" chains of PTB7 is not well understood. They show a relatively low degree of $\mathrm{x}$-ray scattering, ${ }^{[21]}$ indicating little order, but are not amorphous either. Such "disordered aggregates" are difficult to examine, as a lack of periodicity combined with a complex organization of material leads to few easy ways to characterize them.

Here we have extended our single molecule fluorescence studies on PTB7 to observe the dynamical nature of the emission polarization, in terms of linear dichroism, and photoluminescence spectrum of isolated single chains/aggregates of chains. By simultaneously measuring both the time dependence of the emission color and linear dichroism of the chains we can examine how the active emitting units change over longer time periods, and extract some understanding of the nature of disordered aggregates in PTB7. We find high frequency fluctuations in both the emission polarization and spectrum, which can exist inside otherwise apparently stable aggregates. Identifiable jumps in either observable can occur and can be correlated with other photophysical properties, such as the photoluminescence (PL) lifetime, to tell us something about how the excited states of the material evolve over longer periods of time. Finally, examples are shown where direct correlations are observed between the linear dichroism and the emission energy, and this is used to reconstruct two possible arrangements of the PTB7 chains that are studied. 


\section{Results \& Discussion}

The chemical structure of PTB7 along with the ensemble absorption spectrum and two typical single molecule photoluminescence (PL) spectra reported in our previous investigations ${ }^{[18]}$ are shown in Figure 1a. Shown in Figure 1b is the detection schematic used for this study and the equations used to calculate the variables of relevance, the linear dichroism (LD) and the color ratio centered at $646 \mathrm{~nm}\left(\mathrm{R}_{\mathrm{RG}}\right)$, as discussed above. The key point to note is that both variables will lie between -1 and +1 . For an individual spot the absolute LD does not tell us anything about the orientation of the emission dipole, but $\Delta \mathrm{LD}$ will enable us to comment on the change in emission dipole angle as a function of time for a single spot. For $\mathrm{R}_{\mathrm{RG}}$ we can tell where the PL spectrum likely sits, with the green PL spectrum in Figure 1a representing a 50:50 balance between the detectors and thus $\mathrm{R}_{\mathrm{RG}} \sim 0$, while the red PL spectrum will give $\mathrm{R}_{\mathrm{RG}} \sim 1$. 

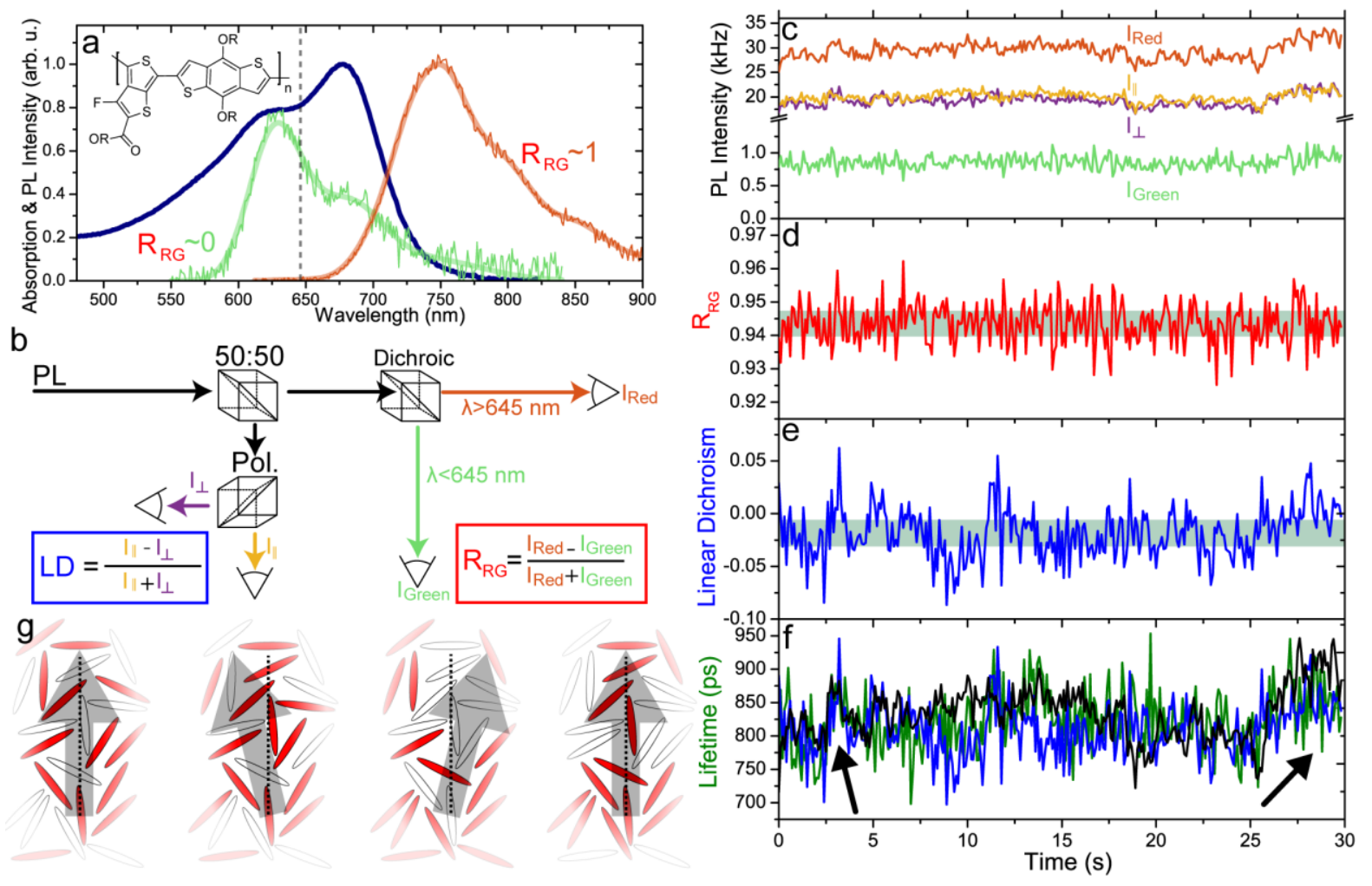

Figure 1. Shown in (a) is the absorption (navy line), red aggregate PL (red line) and green straight-chain PL (green line) spectra of PTB7 from our previous publication, ${ }^{[18]}$ with the chemical structure inset, where $\mathrm{R}=2$-ethylhexyl. In (b) the detection schematic used with the microscope is shown, where PL is split 50:50 between linear dichroism (LD) and red fraction $\left(\mathrm{R}_{\mathrm{RG}}\right)$ arms, with the equations used to define both variables boxed in blue and red respectively. The PL intensity on each detector (c), ratio of red to green emission, $\mathrm{R}_{\mathrm{RG}}(\mathbf{d})$ and LD (e) of a single PTB7 aggregate observed for $30 \mathrm{~s}$ measurement time and binned at $100 \mathrm{~ms}$ resolution are shown. In (f) the PL lifetime (green line) compared with the total PL intensity (black line) and LD (blue line) are shown for the same aggregate, with the arrows indicating regions where strong correlations are observed between these three parameters. The green area in both $R_{R G}$ and LD plots ( $\mathrm{d}$ and $\mathrm{e}$ ) indicate the expected $1 \sigma$ standard deviation resulting from the detectors, showing that while $\mathrm{R}_{\mathrm{RG}}$ fluctuations broadly lie within this noise level, larger fluctuations are observed in the LD that must originate from fast fluctuations in the emission dipole orientation, rather than noise on the detectors. Finally, in (g) a schematic of the likely variation in emitting chromophores (shaded red) as a function of time is shown, along with the overall emission dipole orientation (grey arrow), indicating that while the emission spectrum changes little (as all chromophores are red), the effective emission dipole across a number of chromophores varies as a function of time.

\subsection{Fast Fluctuations of Observables}

First, as shown in Figure 1c-f, we can look at a single bright red aggregate of PTB7. Here the PL signals from the 4 detectors are shown in panel c, while the corresponding $\mathrm{R}_{\mathrm{RG}}$ and $\mathrm{LD}$ are shown in panels $\mathrm{d}$ and e respectively. All data is binned at a $100 \mathrm{~ms}$ time resolution. The $\mathrm{R}_{\mathrm{RG}}$ has a mean value of $\sim 0.944$, indicating that this is a completely red spot, characteristic of a 
disordered aggregate of a significant number of chain segments. The mean LD is around -0.02 , and as noted above, the absolute LD does not tell us anything as the orientation of the individual chromophore(s) with respect to the detection axes. However, our interest is not in the static mean values for these variables, but any dynamical fluctuations in them as a function of the measurement time. To help distinguish fluctuations the shaded green regions in the $\mathrm{R}_{\mathrm{RG}}$ and LD data indicate the $1 \sigma$ standard deviation calculated from the raw signals on the two detectors that the $\mathrm{R}_{\mathrm{RG}}$ and $\mathrm{LD}$ values derive from, taking into account error propagation through the equation and assuming shot noise on the detectors. ${ }^{[22]}$ Therefore the green shaded area is in effect the $1 \sigma$ confidence interval for the $\mathrm{R}_{\mathrm{RG}}$ and $\mathrm{LD}$ that we would expect solely from detector noise, thus any variation significantly greater than this must be due to fluctuations in the $R_{R G}$ or LD value from the spot as a function of time, rather than simply detector noise. It can be seen that while the $\mathrm{R}_{\mathrm{RG}}$ values broadly sit within or on the $1 \sigma$ confidence limit, the LD values vary more significantly. This tells us that while the emission spectrum of the red aggregate does not blue-shift in any meaningful way, the excitons are not static in place on such an aggregate over this $30 \mathrm{~s}$ measurement window, with the polarization jumps indicating that the overall effective emission dipole orientation is fluctuating.

We can to some extent quantify the polarization fluctuations if we were to look at the overall change in LD over the duration of the trace. Taking the approximate highest and lowest LD values $(+0.05$ and -0.075$)$ we obtain $\Delta \mathrm{LD}=0.125$, which corresponds to an angle change of either $\sim 8^{\circ}$ or $\sim 172^{\circ}$, however the former seems much more likely. As this is a bright red aggregate, we can ascertain that it will comprise of a number of concurrently emitting chromophores. This is determined from the photon antibunching, ${ }^{[23-26]}$ where we use a Hanbury Brown and Twiss (HBT) arrangement, ${ }^{[27-29]}$ to measure the cross-correlation between two channels. The existing data can be reconstituted into an HBT arrangement by merging the two LD channels into one, and the two $R_{R G}$ channels into one, giving us a classic 50:50 split of 
the PL. These two merged photon streams can then be cross-correlated with each other to obtain information on photon antibunching which will tell us about the number of emitters in the spot. ${ }^{[30]}$ For this red aggregate we find that between 5 and 10 emitters are present (as shown in the Supporting Information), which is also consistent with our previous observations. ${ }^{[18]}$ Consequently we can build a picture (Figure 1g) of what the LD fluctuations may actually represent. Different red aggregated chromophores switch on and off such that, as a function of lab time, the total effective LD across all emitting chromophores varies, as shown with the large grey arrow. The overall observed PL spectrum does not change (because all chromophores are red), but as different chromophoric sites are activated and deactivated we do see these changes in the LD that leads to the effective variation of $\sim 8^{\circ}$ in the emission dipole. We see no clear periodicity or deterministic behavior of the switching between chromophores, with the variation in LD appearing to be essentially random as a function of time. Most likely highly localized effects are controlling this switching, e.g. as a chromophore temporarily bleaches then one nearby (with a different orientation) is able to instead act as the emission site, but the bleach and its recovery are all randomly occurring in time.

We now examine whether any of these fluctuations can be correlated with other measurable photophysical parameters. It is clear there is no correlation between the $\mathrm{LD}$ and $\mathrm{R}_{\mathrm{RG}}$. This lack of correlation does not exclude a spectral correlation, it is just that the dichroic used to determine the parameter $R_{R G}$ is sensitive to the wavelength region $\sim 646 \mathrm{~nm}$, so if there is no PL around this wavelength then the $\mathrm{R}_{\mathrm{RG}}$ will not show any changes. However, we do see a correlation between the LD, the fitted PL decay time constant and the overall PL intensity, as shown in Figure 1f. While there is no perfect correlation, there are clear points where all observables show the same response, indicated in the panel with arrows. Working with fast fluctuations makes this effect hard to explore in more detail, so we thus looked for example 
spots that showed single step changes in the observed photophysics to allow further information to be extracted.

\subsection{Single Step Changes in Observables}
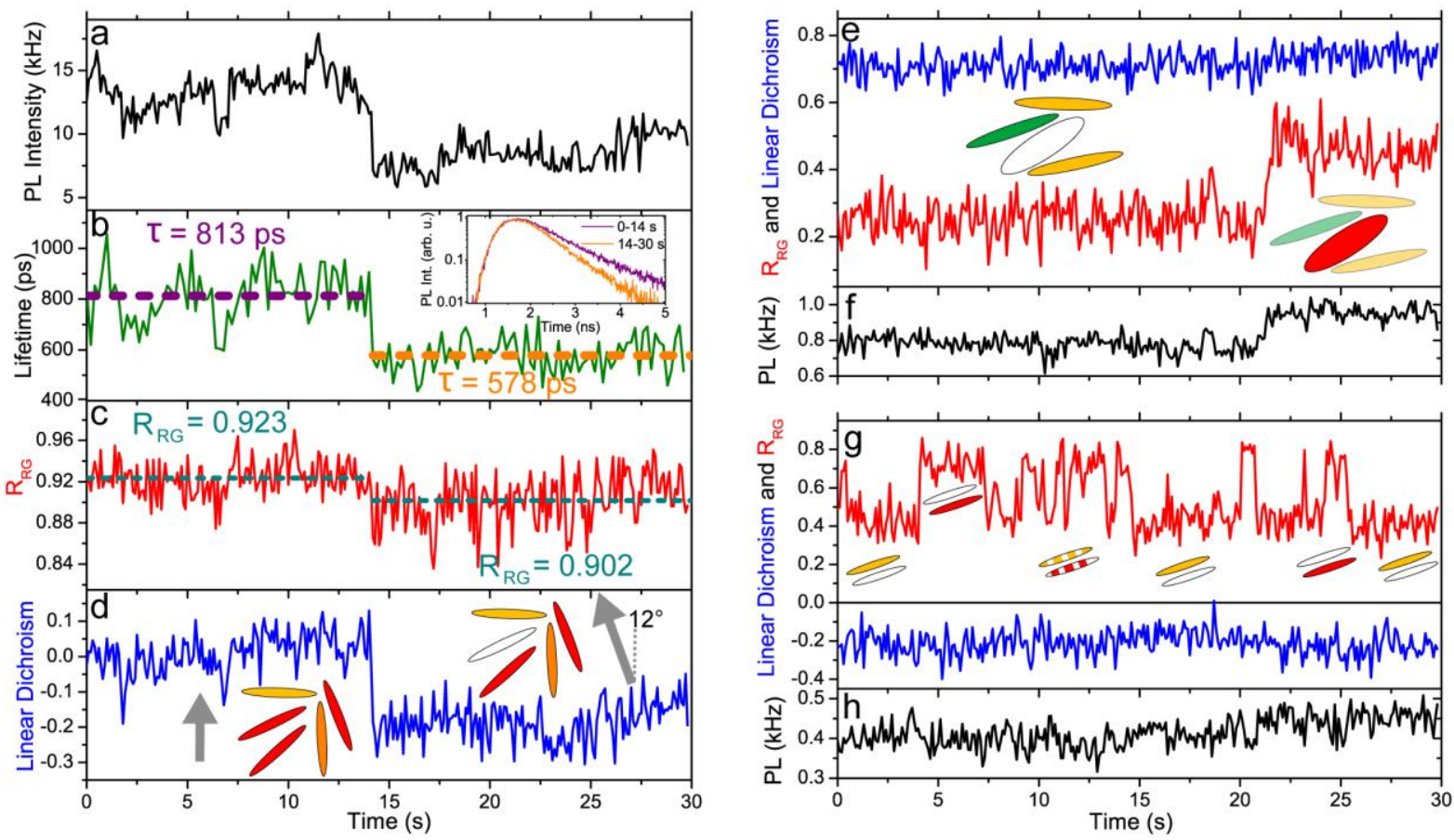

Figure 2. The total PL intensity (a), fitted decay time constant (b), ratio of red to green emission, $\mathrm{R}_{\mathrm{RG}}(\mathbf{c})$ and linear dichroism (d) of a single PTB7 aggregate observed for $30 \mathrm{~s}$, binned at $100 \mathrm{~ms}$ resolution (200 ms for the lifetime data). A large drop in the total PL intensity at a time of $14 \mathrm{~s}$ is mirrored in a drop in the PL lifetime, with the average lifetime in the 0-14 and 14-30 s windows indicated by the dashed lines, with the lifetimes, tau, stated and the PL decays measured in the corresponding time windows inset in panel b. The drop in intensity is also mirrored with a large change in the LD, corresponding to a $12^{\circ}$ angle change, and a small change in the $R_{R G}$ value, with the average value in the two time windows indicated by the solid lines and stated numbers. A schematic of the changes that occur at $14 \mathrm{~s}$ is shown in the inset of $\mathrm{d}$, with the grey arrows representing an effective emission dipole orientation that changes by $12^{\circ}$ at $14 \mathrm{~s}$. In (e) and (g) two further PTB7 spots with step-like changes of observables are shown, here with large jumps in $\mathrm{R}_{\mathrm{RG}}$ and relatively stable $\mathrm{LD}$ values. Corresponding traces for the total PL intensity are shown in (f) and (h), with a clear rise in intensity seen in (f) correlating to the change in $R_{R G}$, while no changes are seen in the intensity for $(h)$. Schematics are shown in the inset for the likely nanoscopic picture of what may be occurring to give rise to these observed jumps.

Dynamical information in isolated PTB7 chains that exhibit single-step changes in their photophysical properties is shown in Figure 2. First, in panels a-d we examine a single red emitting aggregate that undergoes significant changes after $14 \mathrm{~s}$ of measurement. The overall 
PL intensity drops, the extracted PL lifetime drops, $\mathrm{R}_{\mathrm{RG}}$ drops by a small amount and the LD changes by a significant amount. Photon antibunching tells us that there is more than one emitting chromophore (likely between 2 and 4) present in this aggregate (see Supporting Information), so we should consider the change at $14 \mathrm{~s}$ with respect to the nature of multichromophoric emission. Unfortunately the signal levels of the recorded photon statistics do not allow us to determine the number of emitters before and after the 14 second mark, but given the large drop in PL intensity it is likely that we have reduced the number of active emitters, an explanation that is consistent with previous single molecule observations in P3HT. ${ }^{[31]}$ The change in the fitted natural lifetime at 14 seconds likely embodies a number of processes that are difficult to disentangle. That we see a significant drop in the lifetime tells us that that the effective (a weighted average across each of the emissive chromophores) radiative and nonradiative decay rates from the first excited state have changed, but we are unable to comment further as the drop in PL intensity will be due to both a combination of the change in the rates but also a drop in the number of active emitters. When we also add in the information we have on the $\mathrm{R}_{\mathrm{RG}}$ and LD dynamics (panels $\mathrm{c}$ and $\mathrm{d}$ ) then we can gain more insight into what happens. There is a subtle change in $\mathrm{R}_{\mathrm{RG}}$ at $14 \mathrm{~s}$ (the dashed lines show the average values before and after $14 \mathrm{~s}$, with numbers as stated), and a much larger change in the LD, equivalent to an almost $12^{\circ}$ change in the effective emission dipole orientation (again, we discount the possible solution corresponding to a large change in angle, which here would be $168^{\circ}$ ). Both of these changes are consistent with the hypothesis of a bleaching of one of the emitters in the multichromophoric aggregate occurring at $14 \mathrm{~s}$. Overall a picture of what is happening is sketched in the inset of panel d. From 0-14 s we have a collection (>2) of emitting chromophores. These are likely to be non-interacting as otherwise they would funnel excitation energy to each other, giving rise to exciton-exciton annihilation, but we have drawn them next to each other here in the sketch for simplicity. The collection of chromophores has an effective LD (shown as the 
grey arrow), an overall $R_{R G}$ that derives from primarily red but a few greener emitters, and lifetime derived from a weighted average of each constituent chromophore. At $14 \mathrm{~s}$ a bleach of a fully red chromophore stops it from absorbing and emitting, decreasing the overall $R_{R G} a$ little, changing the average radiative and non-radiative rates and leading to a $12^{\circ}$ change in the effective orientation of emission. An expansion of this methodology would be to try and bleach out further chromophores until we are left with just one. That would allow us to reconstruct the full organization of the aggregate by working backwards from one to many, deducing each chromophore's color/orientation as we go.

On the right hand side of Figure 2 (panels e to h) we examine two single chains of PTB7 that undergo step-changes to their emission spectrum as a function of time. In the first (panels e and f) a single change occurs at time $21 \mathrm{~s}$ with $\mathrm{R}_{\mathrm{RG}}$ increasing from $\sim 0.2$ to $\sim 0.6$, i.e. significant red-shifting of the PL. There is also an increase in the total PL intensity at the same time; however there is only a small and very subtle increase in the LD. Again, photon statistics indicate that there is more than one emitting chromophore in this spot (see Supporting Information), but a definite number of chromophores cannot be confidently assigned owing to low signal levels. Overall the change at $21 \mathrm{~s}$ is suggested to be the "switching on" of a red chromophore, as depicted in the sketch in panel e, with the emission dipole orientation being similar to that of the existing chromophores. This single-step color change contrasts with another PTB7 spot, shown in Figure 2 panels $g$ and h, that displays continual color switching between an $R_{R G}$ of 0.4 and 0.8 , i.e. orange and red. No commensurate changes in LD or PL intensity are observed, indicating that we have chromophores that are well aligned with each other and that one switches on only when the other switches off (if one was always on and the other switched on-off-on we should observe a variation in the PL intensity). Unfortunately the PL count rate is too low to extract any meaningful photon statistics to tell us about the number of chromophores, but having specifically one active chromophore would be consistent with the 
switching behavior. The switching is clear and fast, and shows two forms: long dwell-time periods (e.g. from 0 to 4 and 4 to $7.5 \mathrm{~s}$ ) and short fast cycling (e.g. in the 10 to $15 \mathrm{~s}$ window). Inset in the plot of the $\mathrm{R}_{\mathrm{RG}}$ data is a color coded chromophore schematic to indicate this behavior, with aligned chromophoric sites that switch between each other.

In the $\mathrm{LD}$ and $\mathrm{R}_{\mathrm{RG}}$ step changes that are shown in Figure 2 the most striking observation is the degree of changes evident in a 30 s measurement window. PTB7 chains can show remarkable dynamical fluctuations in their PL spectra or emission dipole orientation as a function of time. The multi-chromophoric nature of these disordered aggregates enhances and supports this behavior. Fluctuations in the emission spectrum and polarization have previously been observed at low ${ }^{[32-35]}$ and at room temperature ${ }^{[36]}$ in single molecules. When the molecule only supports one chromophore this behavior is most often assigned at room temperature to variation in the nuclear coordinate as a result of vibrational energy deposited into the molecule and the surrounding bath matrix, particularly when non-radiative excited-state relaxation occurs. In PTB7, however, we most likely have a number of chromophoric sites, of varying energy and orientation. As a function of time, therefore, the excited state can "sample" some of this energetic and orientational landscape, tracing out a picture for us of how these chromophores are arranged. This conclusion is evidence of the power of this technique to understand the complex arrangement and aggregation of conjugated polymers. Much work has been expended on investigating the electronic, physical and morphological arrangement of many conjugated polymers including PPV-based materials ${ }^{[33,34,37-46]}$ and P3HT. ${ }^{[47-52]}$ What emerges from these studies is that even at the single chain level, the spatial-energetic arrangement of chains is not always well understood. This question is of strong relevance for new low bandgap conjugated polymers used in organic photovoltaics, where such material arrangements can be crucial to photovoltaic performance, with single molecule studies now helping to understand the photophysics and spatial organization of materials such as PTB7 ${ }^{[18]}$ and PCDTBT. ${ }^{[53]}$ 


\subsection{Correlated Emission Wavelength and Linear Dichroism}

Finally, we wish to highlight PTB7 spots that show an impressive degree of correlation between dynamics in their emission energy and the polarization of that emission. Shown in Figure 3 are three spots (a-c) that display a high degree of such correlation.

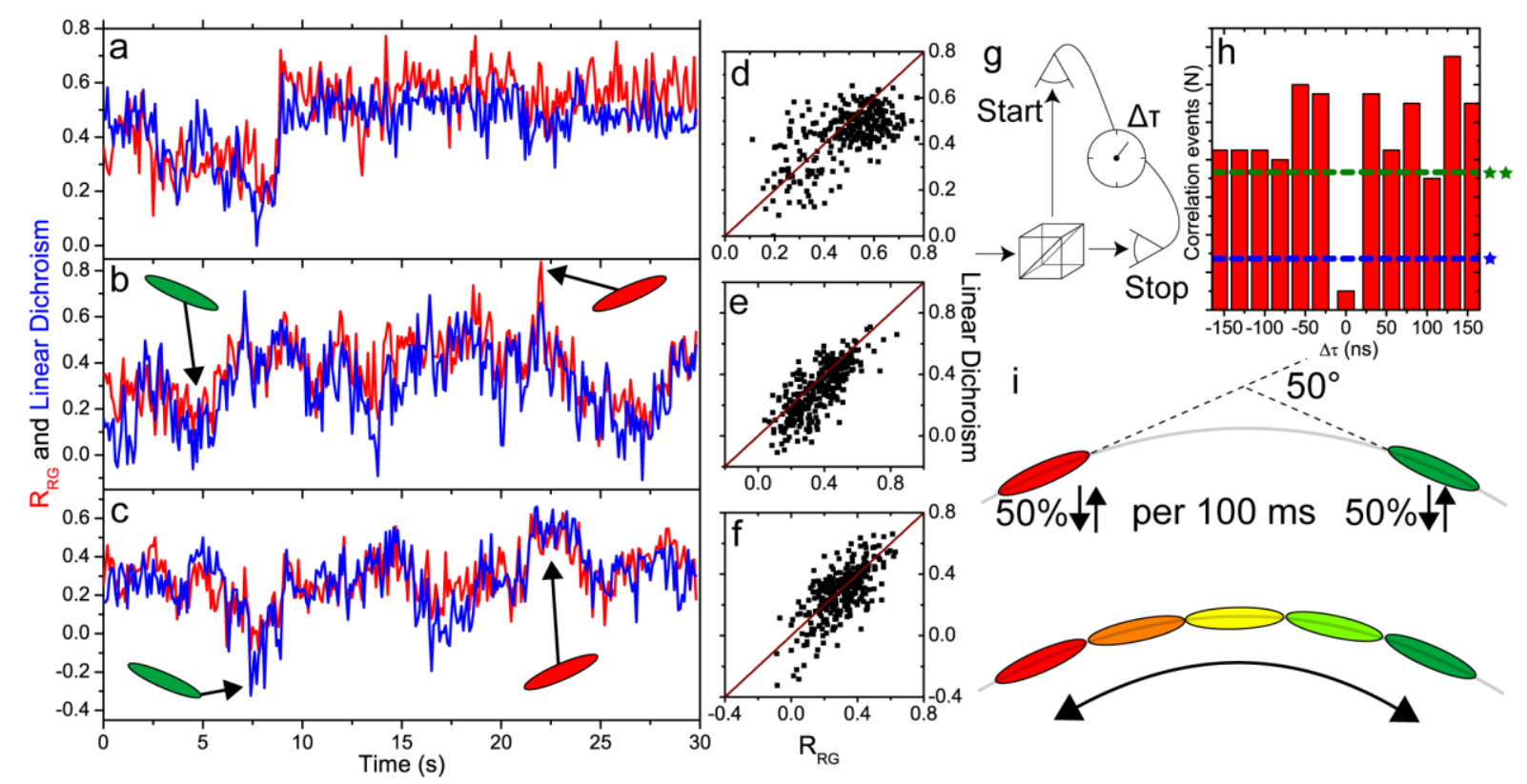

Figure 3. Examples of $R_{R G}$ (red line) and $L D$ (blue line) dynamics for three spots (a-c) that show almost perfect correlation between the two values across a $30 \mathrm{~s}$ window, with data binned at $100 \mathrm{~ms}$ resolution. Correlation plots of $L D$ vs $R_{R G}(\mathbf{d}-\mathbf{f})$ are shown for the same three spots, with the red line indicating the $\mathrm{x}=\mathrm{y}$ diagonal, suggesting that all three fluorescence spots exhibit a strong degree of correlation between LD and $\mathrm{R}_{\mathrm{RG}}$. The Hanbury Brown \& Twiss arrangement of detectors to measure photon antibunching is shown in $\mathbf{g}$, while the antibunching of four PTB7 spots that exhibit strong LD vs $R_{R G}$ correlation (three from this figure and a further one) is shown in $\mathbf{h}$. A strong antibunching dip is observed, with lines indicating the expected dip for one and two emitters, suggesting that there is only one emitting chromophore active at a time in these systems. In (i) two possible schematics for chromophore arrangements are shown, as discussed in the text.

The absolute values of $\mathrm{R}_{\mathrm{RG}}$ and LD match each other almost exactly and they show a very high degree of correlation as each fluctuates. What this correlation means in plain terms is that as the emission spectrum of a PTB7 spot changes, its polarization changes at exactly the same rate. This is easily shown if we plot the $L D$ vs $R_{R G}$ for each spot (panels d-f), where the individual $100 \mathrm{~ms}$ binned points lie on the $\mathrm{x}=\mathrm{y}$ diagonal. To learn about the number of emitting chromophores, we can measure the photon antibunching. The low PL quantum yield of PTB7 
of $\sim 2 \%{ }^{[54]}$ leads to low observed PL counts, thus it is extremely hard to acquire sufficient photon coincidences from these correlated spots, but we can nevertheless comment on the number of emitters. This is done for the three spots shown in Figure 3 and an additional spot that also showed a $\mathrm{R}_{\mathrm{RG}}-\mathrm{LD}$ correlation, and the summated results are shown in Figure $3 \mathrm{~h}$. What is clear is that despite the relatively low signal to noise, a strong and almost perfect photon antibunching dip is observed at zero delay between the channels, indicating that only a single emitter is present in these correlated spots at any one time. This fact has to be reconciled with the continual correlated change in $\mathrm{R}_{\mathrm{RG}}$ and $\mathrm{LD}$ that is measured. The total $\Delta \mathrm{LD}$ for the spots in panels $\mathrm{b}$ and $\mathrm{c}$ is $\sim 0.9$, giving an angle change of $\sim 50^{\circ}$ (here the other solution, $130^{\circ}$, is viable but for simplicity we stick with the smaller angle change), while the $\mathrm{R}_{\mathrm{RG}}$ varies from $\sim 0$ (green spectrum) to $\sim 0.6-0.8$ (orange to red spectrum). There are two possible ways that such correlated continual change could occur, shown schematically in Figure 3i. Either there exist two chromophoric sites, green and red, at an angle of $\sim 50^{\circ}$ to each other and upon each laser pulse only one is excited. When binned on a $100 \mathrm{~ms}$ time window we then observe an average of the selection between these two chromophores, and this average varies as a function of lab time over the $30 \mathrm{~s}$ measurement duration. Alternatively, there could be a number of chromophores of varying optical gap, with the energy of the chromophore matched to a specific angle change, and these are sampled one at a time across the $30 \mathrm{~s}$ measurement, again with $\sim 50^{\circ}$ angle between the extreme red and green sites. We cannot say which of these two possibilities is the more probable, but following Occam's razor the first one is preferred owing to its simplicity.

All of the work detailed here has been pursued with an aim to aspire towards understanding the organization, nature and photophysical properties of disordered aggregates that exist in PTB7. It is worth returning to the importance of this in the context of organic photovoltaic devices, where the red absorption of PTB7 is the key aspect of why it is used to make highly efficient 
solar cells. That absorption derives from the disordered aggregated nature of how the chains organize themselves. Studying such disordered aggregates is very challenging, owing to the small length scales, lack of periodicity and lack of easy tools to controllably separate or aggregate the chains (e.g. with thermal or solvent assisted annealing ${ }^{[13,15]}$ ). In the work presented here we have been able to look at these aggregates of PTB7 and monitor how their emission spectrum and polarization change as a function of time. This has been able to tell us that far from being static emitters, the excited state is constantly changing both its emission energy and orientation. In doing this it can effectively map out a broad range of properties as the excited state inhabits different chromophores in the aggregate. As a proof of concept we have been able to qualifiably examine the nature and degree of spectral and polarization changes that exist, finding fast fluctuations, single step jumps that correlate with changes in lifetime and intensity, temporary jumps and finally correlated jumps between both the emission spectrum and polarization.

\section{Conclusion}

In this work we have investigated the dynamical fluctuations in the PL wavelength and emission dipole orientation in single chains and aggregates of the high performance organic photovoltaic polymer PTB7. We find that rather than always being constant and static, there can be a large degree of fluctuations in both variables, indicative of the complex underlying spatial and electronic organization of the chains. It is ordinarily presumed that a collection of material will reliably behave the same way when it is tested repeatedly, but that is not the case here. The highly disordered and complex arrangement of PTB7 chains can lead to fluctuations in the favored sites for PL to originate from. Most of the examples reported here have been to explore the different ways that these fluctuations can occur, e.g. a change in emission polarization but not wavelength or vice versa, fast fluctuations in emission wavelength, or correlated changes between polarization and wavelength. These changes have been linked to at least a basic schematic of the likely organization of the underlying chromophores in the 
PTB7 chain or aggregate. To realize the vision of fully mapping the spatial and energetic arrangement of chains in disordered aggregates such as those found in PTB7, new studies can be proposed that measure this in detail, working with candidate single aggregates to sequentially bleach chromophores allowing a full spatial-energetic picture of that aggregate to be reconstructed. This concept would be crucial in trying to bridge the gap between understanding of highly ordered organic photovoltaic materials (e.g. P3HT) and amorphous ones (e.g. MEH-PPV), as the disordered but aggregate nature of materials like PTB7 is crucial in enabling its photovoltaic performance, and cannot be fully understood with ensemble structural analysis techniques such as x-ray or neutron diffraction.

\section{Experimental Section}

Materials: $\quad$ Poly[[4,8-bis[(2-ethylhexyl)oxy]benzo[1,2-b:4,5-b']dithiophene-2,6-diyl][3fluoro-2-[(2-ethylhexyl)carbonyl]thieno[3,4-b]thiophenediyl]], substantially and conveniently shortened to the acronym PTB7, was purchased from Sigma Aldrich and used without further purification. The material was dissolved in spectrophotometric grade chlorobenzene and then diluted down to single molecule concentrations before being mixed with a complementary poly(methyl-methacrylate) (PMMA) solution that was used to spin coat films of $\sim 100 \mathrm{~nm}$ thickness onto cleaned coverslip glass. All material and sample preparation was completed in a nitrogen filled glovebox. The sample that was measured had clearly separated diffraction limited spots, with a range of photoluminescence (PL) spectra and degrees of aggregation as we reported previously. ${ }^{[18]}$ The sample was measured under a constant nitrogen flow on the microscope to prevent photobleaching due to oxygen..

Microscopy measurements: Single molecule spectroscopy was performed on a homebuilt confocal microscope. Excitation was provided by a $485 \mathrm{~nm}$ picosecond laser diode operated at $80 \mathrm{MHz}$ (PicoQuant, LDH-D-C-485). The excitation beam was spectrally cleaned, spatially expanded and coupled into an Olympus IX71 microscope base. An Olympus, UPLSAPO 
$60 \mathrm{XO}, \mathrm{NA}=1.35$ objective was used to focus the light and collect PL, which was brought out of the microscope, fed through a pinhole and then onto the detection system as described below and shown in Figure 1b. The sample was moved with a Physik Instrumente P-527.3CL piezo stage.

Two properties, the linear dichroism (LD) and the red to green color ratio $\left(\mathrm{R}_{\mathrm{RG}}\right)$ were simultaneously recorded. This was achieved by splitting the PL collected with the confocal microscope with a 50:50 cube. Half of the PL went to the LD detection arm where the PL was split with a polarizing cube into PL parallel and perpendicular to an arbitrary optical axis and detected on two avalanche photodiodes (APDs, Laser Components COUNT-T100). The other half of the PL was directed to the $\mathrm{R}_{\mathrm{RG}}$ arm, where a dichroic beam splitter (AHF Analysentechnik F33-646) centered at $646 \mathrm{~nm}$ split the PL based on its wavelength and was again detected on two APDs (PicoQuant, $\tau$-SPAD-20). The properties measured with this setup can be simplified to the variables $\mathrm{LD}$ and $\mathrm{R}_{\mathrm{RG}}$ (both defined by the boxed equations in Figure 1b). Spots are measured for 30 seconds and signals from all four APDs are fed into Picoquant HydraHarp 400 time correlated single photon counting (TCSPC) electronics. Piezo stage control and TCSPC instrument control are enabled with homecoded LabView scripts. Data is analysed with homecoded Matlab scripts.

\section{Supporting Information}

Supporting Information is available from the Wiley Online Library or from the author. 


\section{References}

[1] J. D. Chen, C. H. Cui, Y. Q. Li, L. Zhou, Q. D. Ou, C. Li, Y. F. Li, J. X. Tang, Adv. Mater. 2015, 27, 6.

[2] D. Deng, Y. Zhang, J. Zhang, Z. Wang, L. Zhu, J. Fang, B. Xia, Z. Wang, K. Lu, W. Ma, Z. Wei, Nat. Commun. 2016, 7, 13740.

[3] W. Zhao, S. Li, H. Yao, S. Zhang, Y. Zhang, B. Yang, J. Hou, J. Am. Chem. Soc. 2017, 139, 21.

[4] S. Zhang, Y. Qin, J. Zhu, J. Hou, Adv. Mater. 2018, 30, 20.

[5] G. J. Hedley, A. Ruseckas, I. D. W. Samuel, Chem. Rev. 2017, 117, 2.

[6] T. M. Clarke, J. R. Durrant, Chem. Rev. 2010, 110, 11.

[7] M. Stephen, K. Genevicius, G. Juska, K. Arlauskas, R. C. Hiorns, Polym. Int. 2017, $66,1$.

[8] A. Pivrikas, N. S. Sariciftci, G. Juska, R. Osterbacka, Prog. Photovoltaics 2007, 15, 8.

[9] P. F. Barbara, A. J. Gesquiere, S. J. Park, Y. J. Lee, Acc. Chem. Res. 2005, 38, 7.

[10] D. Woll, E. Braeken, A. Deres, F. C. De Schryver, H. Uji-i, J. Hofkens, Chem. Soc. Rev. 2009, 38, 2.

[11] J. M. Lupton, Adv. Mater. 2010, 22, 15.

[12] B. Kozankiewicz, M. Orrit, Chem. Soc. Rev. 2014, 43, 4.

[13] J. Vogelsang, T. Adachi, J. Brazard, D. A. V. Bout, P. F. Barbara, Nat. Mater. 2011, $10,12$.

[14] J. Vogelsang, J. Brazard, T. Adachi, J. C. Bolinger, P. F. Barbara, Angew. Chem. Int. Ed. 2011, 50, 10.

[15] J. Vogelsang, J. M. Lupton, J. Phys. Chem. Lett. 2012, 3, 11.

[16] Y. Y. Liang, Z. Xu, J. B. Xia, S. T. Tsai, Y. Wu, G. Li, C. Ray, L. P. Yu, Adv. Mater. 2010, 22, 20. 
[17] X. H. Ouyang, R. X. Peng, L. Ai, X. Y. Zhang, Z. Y. Ge, Nat. Photonics 2015, 9, 8.

[18] G. J. Hedley, F. Steiner, J. Vogelsang, J. M. Lupton, J. Phys. Chem. Lett. 2017, 8, 15.

[19] M. M. Huo, R. Liang, Y. D. Xing, R. Hu, N. J. Zhao, W. Zhang, L. M. Fu, X. C. Ai, J. P. Zhang, J. H. Hou, J. Chem. Phys. 2013, 139, 12.

[20] F. Bencheikh, D. Duche, C. M. Ruiz, J. J. Simon, L. Escoubas, J. Phys. Chem. C 2015, 119, 43.

[21] M. R. Hammond, R. J. Kline, A. A. Herzing, L. J. Richter, D. S. Germack, H.-W. Ro, C. L. Soles, D. A. Fischer, T. Xu, L. Yu, M. F. Toney, D. M. DeLongchamp, ACS Nano 2011, 5, 10 .

[22] F. Steiner, S. Bange, J. Vogelsang, J. M. Lupton, J. Phys. Chem. Lett. 2015, 6, 6.

[23] H. J. Kimble, M. Dagenais, L. Mandel, Phys. Rev. Lett. 1977, 39, 11.

[24] F. Diedrich, H. Walther, Phys. Rev. Lett. 1987, 58, 3.

[25] T. Basche, W. E. Moerner, M. Orrit, H. Talon, Phys. Rev. Lett. 1992, 69, 10.

[26] L. Fleury, J. M. Segura, G. Zumofen, B. Hecht, U. P. Wild, Phys. Rev. Lett. 2000, 84,

6.

[27] R. H. Brown, R. Q. Twiss, Nature 1956, 177, 4497.

[28] R. H. Brown, R. Q. Twiss, Proc. Royal Soc. Lond. 1957, 242.

[29] R. H. Brown, R. Q. Twiss, Proc. Royal Soc. Lond. 1958, 243.

[30] K. D. Weston, M. Dyck, P. Tinnefeld, C. Muller, D. P. Herten, M. Sauer, Anal. Chem. 2002, 74, 20.

[31] K. S. Grussmayer, F. Steiner, J. M. Lupton, D.-P. Herten, J. Vogelsang, ChemPhysChem 2015, 16, 17.

[32] W. E. Moerner, M. Orrit, Science 1999, 283, 5408.

[33] F. Schindler, J. M. Lupton, J. Feldmann, U. Scherf, Proc. Natl. Acad. Sci. U.S.A. 2004, 101, 41. 
[34] T. Pullerits, O. Mirzov, I. G. Scheblykin, J. Phys. Chem. B 2005, 109, 41.

[35] A. V. Aggarwal, A. Thiessen, A. Idelson, D. Kalle, D. Wursch, T. Stangl, F. Steiner,

S. S. Jester, J. Vogelsang, S. Hoger, J. M. Lupton, Nat. Chem. 2013, 5, 11.

[36] H. P. Lu, X. S. Xie, Nature 1997, 385, 6612.

[37] D. A. VandenBout, W. T. Yip, D. H. Hu, D. K. Fu, T. M. Swager, P. F. Barbara, Science 1997, 277, 5329.

[38] Z. H. Yu, P. F. Barbara, J. Phys. Chem. B 2004, 108, 31.

[39] Y. Ebihara, M. Vacha, J. Phys. Chem. B 2008, 112, 40.

[40] S. Habuchi, S. Onda, M. Vacha, Phys. Chem. Chem. Phys. 2011, 13, 5.

[41] T. Huser, M. Yan, L. J. Rothberg, Proc. Natl. Acad. Sci. U.S.A. 2000, 97, 21.

[42] H. Z. Lin, S. R. Tabaei, D. Thomsson, O. Mirzov, P. O. Larsson, I. G. Scheblykin, J. Am. Chem. Soc. 2008, 130, 22.

[43] H. Lin, Y. Tian, K. Zapadka, G. Persson, D. Thomsson, O. Mirzov, P.-O. Larsson, J. Widengren, I. G. Scheblykin, Nano Lett. 2009, 9, 12.

[44] A. Kohler, S. T. Hoffmann, H. Bassler, J. Am. Chem. Soc. 2012, 134, 28.

[45] G. A. Sherwood, R. Cheng, T. M. Smith, J. H. Werner, A. P. Shreve, L. A. Peteanu, J. Wildeman, J. Phys. Chem. C 2009, 113, 43.

[46] W. Y. So, J. Hong, J. J. Kim, G. A. Sherwood, K. Chacon-Madrid, J. H. Werner, A. P. Shreve, L. A. Peteanu, J. Phys. Chem. B 2012, 116, 35.

[47] T. Adachi, J. Brazard, R. J. Ono, B. Hanson, M. C. Traub, Z. Q. Wu, Z. C. Li, J. C. Bolinger, V. Ganesan, C. W. Bielawski, D. A. V. Bout, P. F. Barbara, J. Phys. Chem. Lett. 2011, 2, 12.

[48] T. Adachi, G. Lakhwani, M. C. Traub, R. J. Ono, C. W. Bielawski, P. F. Barbara, D. A. Vanden Bout, J. Phys. Chem. B 2012, 116, 32. 
[49] A. Thiessen, J. Vogelsang, T. Adachi, F. Steiner, D. Vanden Bout, J. M. Lupton, Proc. Natl. Acad. Sci. U.S.A. 2013, 110, 38.

[50] F. Steiner, J. Vogelsang, J. M. Lupton, Phys. Rev. Lett. 2014, 112, 13.

[51] F. Steiner, J. M. Lupton, J. Vogelsang, J. Am. Chem. Soc. 2017, 139, 29.

[52] F. Tenopala-Carmona, S. Fronk, G. C. Bazan, I. D. W. Samuel, J. C. Penedo, Sci. $A d v$. 2018, 4, 2.

[53] B. Shao, D. A. V. Bout, J. Mater. Chem. C 2017, 5, 37.

[54] G. J. Hedley, A. J. Ward, A. Alekseev, C. T. Howells, E. R. Martins, L. A. Serrano, G. Cooke, A. Ruseckas, I. D. W. Samuel, Nat. Commun. 2013, 4, 3867. 
Fluctuations in the emission spectrum and polarization of disordered aggregates of PTB7 at single and few chain levels allow reconstruction of the spatial arrangement of chromophores. The nanoscale organization of the chromophores on the polymer chain can be deduced, and fluctuations in the observables recorded as excitons sample different sites on the chain/aggregate.

Single molecule spectroscopy

G.J. Hedley*, F. Steiner, J. Vogelsang, J.M. Lupton

Fluctuations in the Emission Polarization and Spectrum in Single Chains of a Common Conjugated Polymer for Organic Photovoltaics

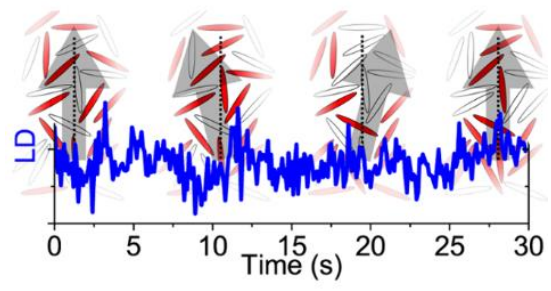




\section{Supporting Information}

\section{Fluctuations in the Emission Polarization and Spectrum in Single Chains of a Common Conjugated Polymer for Organic Photovoltaics}

Gordon J. Hedley, * Florian Steiner Jan Vogelsang and John M. Lupton

\section{Methodology for Photon Antibunching Measurements}

Photon antibunching is measured using a Hanbury-Brown and Twiss arrangement, as described and referenced in the main text. The main difference required here is that after the 50:50 split cube we have a pair of detectors on each arm, 2 to monitor $R_{R G}$ and 2 to measure the linear dichroism. Consequently each 2 detector's counts are merged together to create a single photon stream for each arm of the 50:50, enabling a conventional HBT cross-correlation to be performed. This merging of photon streams is achieved via Matlab scripts, and takes the detected photon's macro and micro times for both channels and orders them together to create a virtual sum channel.

APD afterglow issues with these measurements have led to the first lateral bins $(+-12.5 \mathrm{~ns})$ being discarded. The conventional way to remove afterglow involves using a shortpass filter, but the red emission from PTB7 aggregates precludes that. Consequently discarding the first lateral bins is the safest way to ensure that no afterglow is mistakenly reported. Microtime gating is used on the central bin to ensure that afterglow photons are not counted in the coincidence. We can be confident that no afterglow contributes to the central bin as some antibunching is always observed (and in the case of Figure 3 almost perfectly), which contrasts with the significantly ( $4 \mathrm{x}$ compared to other lateral bins) higher occurrences from afterglow that would definitely eliminate any antibunching dip.

Signal-to-noise is a challenging issue for the reported photon antibunching measurements when we have only 30 second observation windows with low (typically in the $1-15 \mathrm{kHz}$ range) total count rate spread across 4 detectors. Consequently throughout we have re-binned the photon antibunching data down to $40 \mathrm{MHz}$ from the measured $80 \mathrm{MHz}$ (i.e. averaged 2 lateral peaks to get 1). Given the low $\mathrm{S} / \mathrm{N}$ here we are confident that any fast lateral decay (e.g. due to a dark state) will not affect the lateral to central ratio, as such a decay could not be seen with such a low number of correlation events anyway. 


\section{Photon Antibunching for Large Red Aggregate}

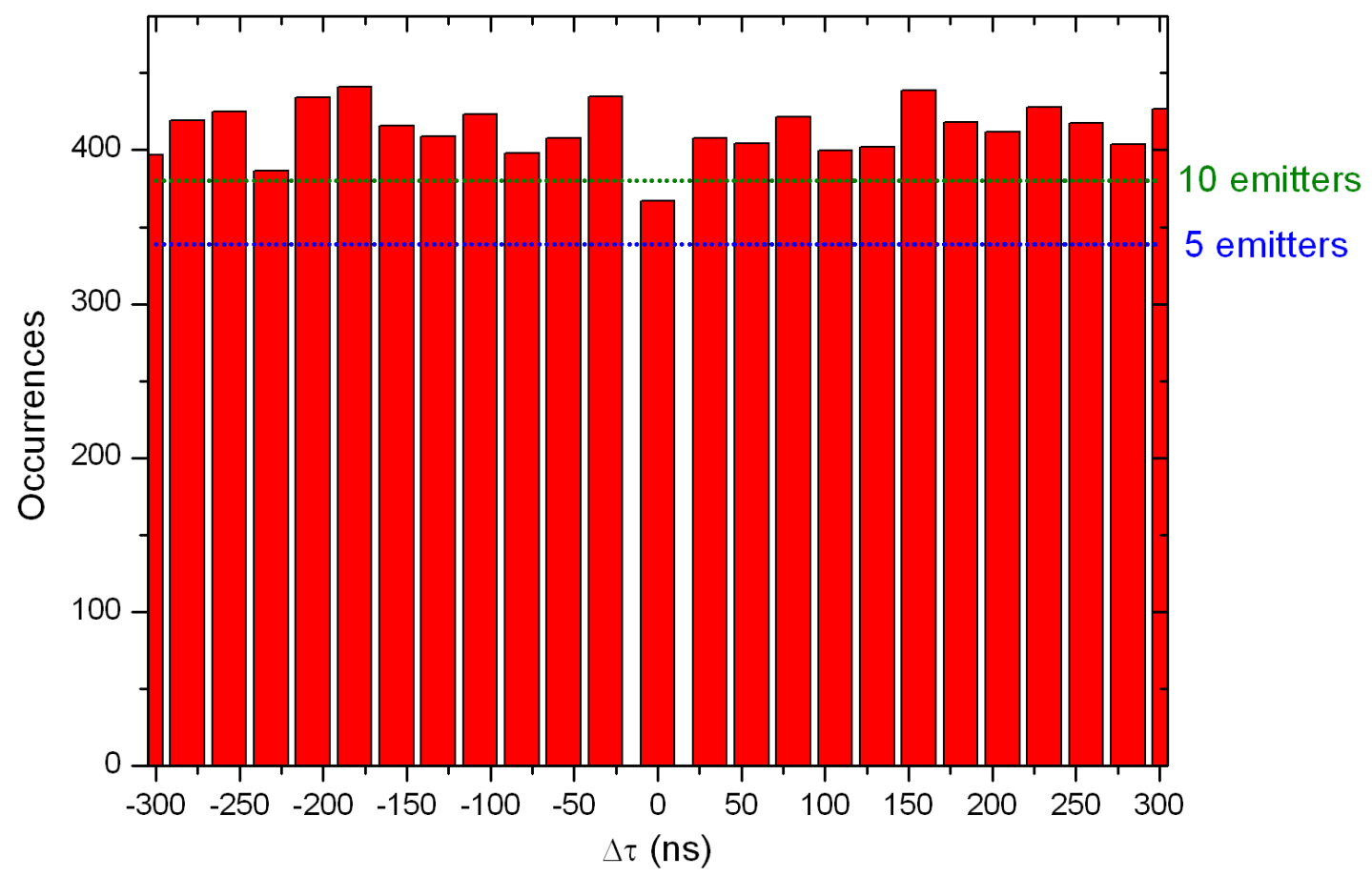

Figure S1: Photon correlation occurrences for the large red aggregate of PTB7 shown in Figure 1 of the main text. The first lag has been removed owing to APD afterglow. The dotted lines indicate the central bin dip that would be expected for 5 and 10 emitters, as per the Weston et al. method referenced in the main text. This indicates that there are between 5 and 10 chromophores in the aggregate. 


\section{Photon Antibunching for Red Aggregate with Step Change in LD at 14 Seconds}

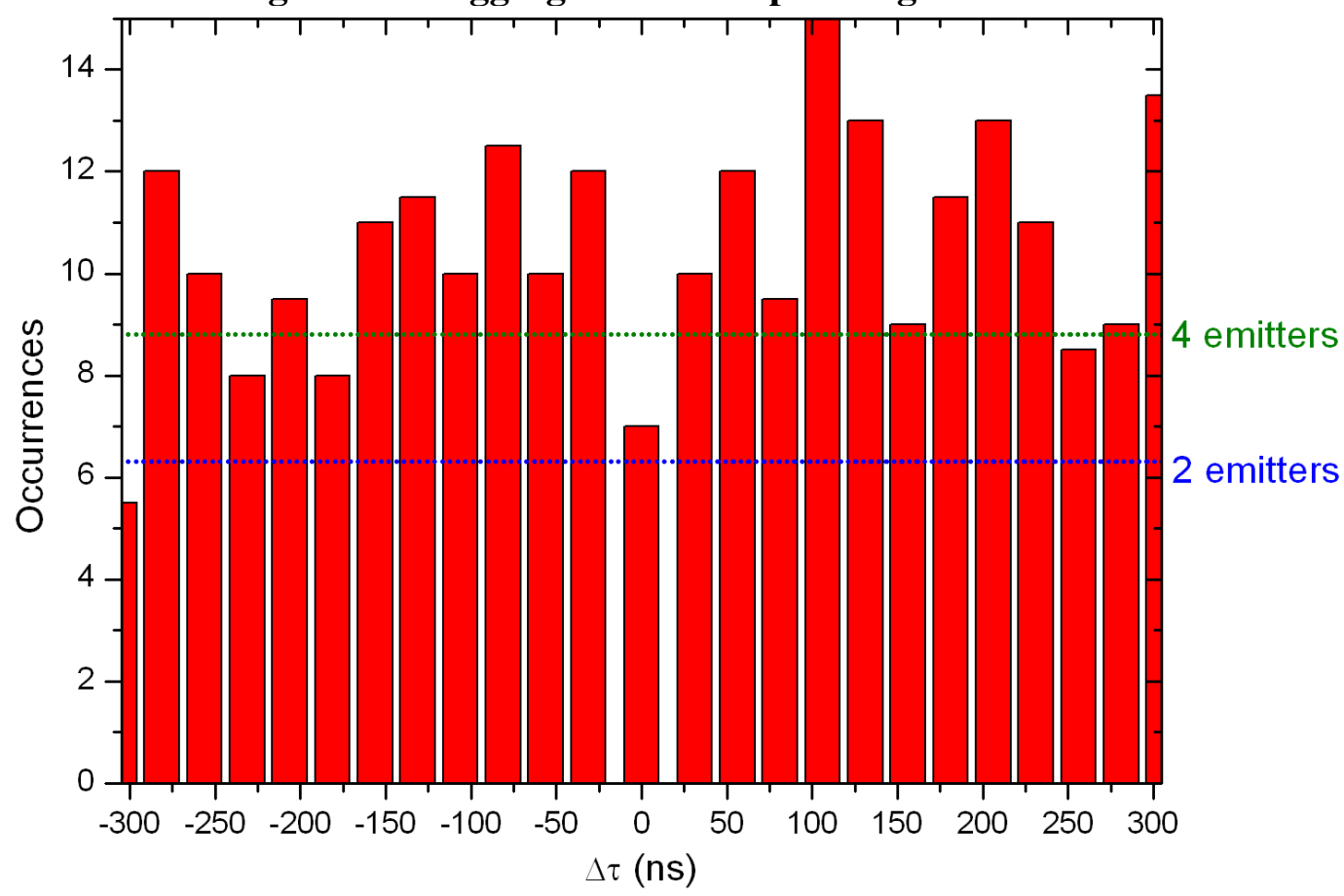

Figure S2: Photon correlation occurrences for the red aggregate of PTB7 shown in Figure 2a$\mathrm{d}$ of the main text that shows a step-change in its LD at 14 seconds. The occurrences shown here are from the entire 0-30 second trace to ensure that there is enough $\mathrm{S} / \mathrm{N}$. The first lag has been removed owing to APD afterglow. The dotted lines indicate the central bin dip that would be expected for 2 and 4 emitters, as per the Weston et al. method referenced in the main text. This indicates that the number of chromophores lies in the range of 2-4. 


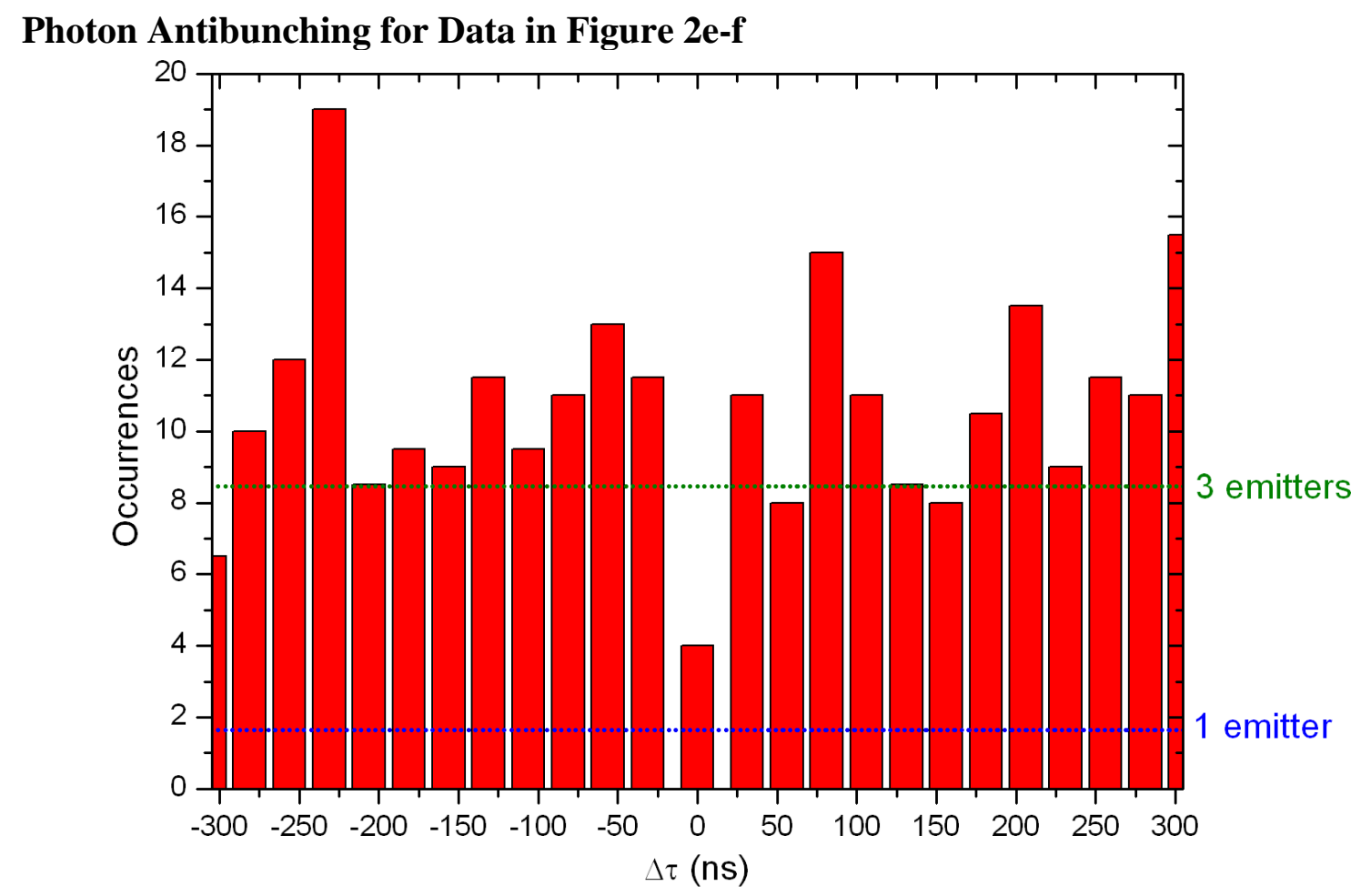

Figure S3: Photon correlation occurrences for the green aggregate of PTB7 shown in Figure $2 \mathrm{e}-\mathrm{f}$ of the main text that shows a step-change in its $\mathrm{R}_{\mathrm{RG}}$ at 21 seconds. The occurrences shown here are from the entire 0-30 second trace to ensure that there is enough $\mathrm{S} / \mathrm{N}$. The first lag has been removed owing to APD afterglow. The dotted lines indicate the central bin dip that would be expected for 1 and 3 emitters, as per the Weston et al. method referenced in the main text. The low signal-to-noise allows us to only really say that there is $>1$ chromophore in the spot. 


\section{Comparison of Red Aggrgate PL Psectra in PMMA and Zeonex Host}

To check that the polar PMMA host matrix was not significantly shifting the PL spectra of the red aggregates, the non-polar host Zeonex was used as an alternative, with no substantive change observed.

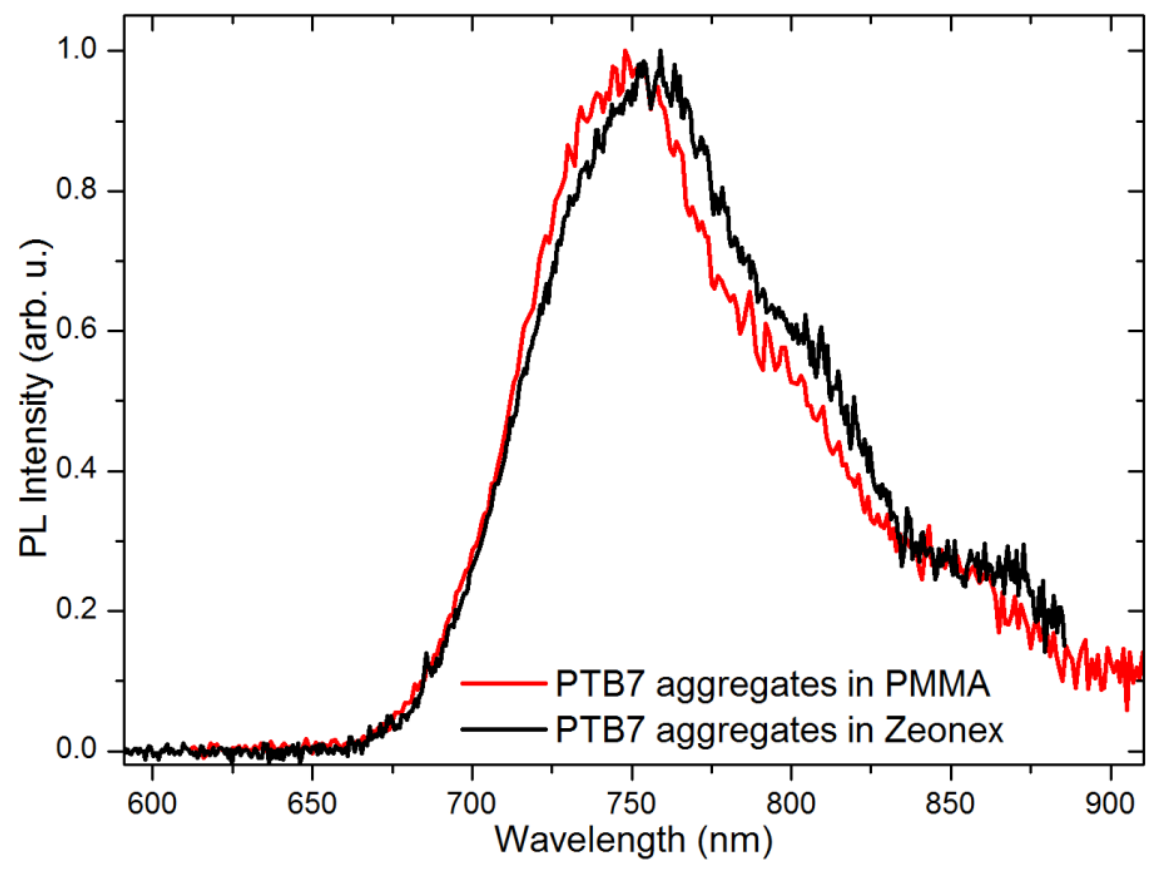

Figure S4: Single aggregate PL spectra of PTB7 in PMMA (red line) and Zeonex (black line) host matricies. 